\title{
Mass loss from dusty, low outflow-velocity AGB stars ${ }^{\star \star \star \star}$ II. The multiple wind of EP Aquarii
}

\author{
J. M. Winters ${ }^{1}$, T. Le Bertre ${ }^{2}$, J. Pety ${ }^{1,2}$, and R. Neri ${ }^{1}$ \\ 1 IRAM, 300 rue de la Piscine, 38406 St. Martin d'Hères, France \\ e-mail: winters@iram.fr \\ 2 LERMA, UMR 8112, Observatoire de Paris, 61 Av. de l'Observatoire, 75014 Paris, France
}

Received 2 July 2007 / Accepted 31 August 2007

\section{ABSTRACT}

\begin{abstract}
Context. CO rotational lines are frequently used to trace the outflows from AGB stars. Some profiles are composite, with a narrow component super-imposed on a broader one. These profiles have been interpreted in different ways, calling for episodic mass loss, a bipolar flow, or a circumstellar disk.

Aims. To investigate the structure of one of these outflows, we have obtained detailed ${ }^{12} \mathrm{CO}(2-1)$ and ${ }^{12} \mathrm{CO}(1-0)$ maps of EP Aqr, a prototypical source with composite $\mathrm{CO}$ profiles.

Methods. Interferometric data were acquired with the IRAM interferometer and combined with on-the-fly maps obtained at the IRAM $30-\mathrm{m}$. The resulting maps in ${ }^{12} \mathrm{CO}(2-1)$ and ${ }^{12} \mathrm{CO}(1-0)$ cover a field of $100^{\prime \prime} \times 100^{\prime \prime}$ with a spectral resolution of $0.1 \mathrm{~km} \mathrm{~s}{ }^{-1}$ and with beams of $1.7^{\prime \prime} \times 1.0^{\prime \prime}$ and $3.5^{\prime \prime} \times 1.8^{\prime \prime}$, respectively.

Results. The source is clearly resolved with a size of about $15^{\prime \prime}(F W H M)$. We do not observe any obvious departure from circular symmetry, but there is evidence of a ringed structure in the $\mathrm{CO}(2-1)$ map with enhanced intensity at $\sim 3.5^{\prime \prime}$ and $7.5^{\prime \prime}$ from the central star. The continuum level at 1.3 and $2.6 \mathrm{~mm}$ is consistent with the star's photospheric emission. We modeled the spatio-kinematic structure with Monte-Carlo radiative transfer simulations assuming spherical symmetry. We reached a reasonable fit to the mapintegrated spectra, but not to the imaging data, possibly because the circumstellar shell of EP Aqr presents inhomogeneities on a scale that is not, or is only barely, resolved in our maps. EP Aqr may be a proto-typical oxygen-rich source for the class of theoretical models exhibiting mass loss variations on a $\sim 100 \mathrm{yr}$ timescale discovered by Winters et al. (2000), which show a layered structure in their extended circumstellar shells.
\end{abstract}

Key words. hydrodynamics - stars: mass-loss - stars: AGB and post-AGB - stars: winds, outflows - radio lines: stars circumstellar matter

\section{Introduction}

Most stars in our Galaxy eventually evolve through the asymptotic giant branch (AGB) and end their lives by stripping off their outer layers in form of a massive molecular wind. One of the best tracers of this phenomenon are the rotational lines of the chemically relatively inert $\mathrm{CO}$ molecule, particularly the $(J=1 \rightarrow 0)$ and $(2 \rightarrow 1)$ lines in the vibrational ground state, which are easily observable from the ground. From a modeling of these lines, mass loss rates have been derived for a large number of AGB stars (e.g., Knapp \& Morris 1985; Kastner 1992; Olofsson et al. 2002) revealing high mass loss rates in the range $\sim 10^{-7}$ and $\sim 10^{-4} M_{\odot} \mathrm{yr}^{-1}$. Modeling of the CO rotational lines is generally considered to be the most reliable method of evaluating the mass loss rates from these late-type stars. However, this approach is usually based on several decisive assumptions; in particular, it assumes i) spherical symmetry of the star and of its circumstellar

* Based on observations carried out with the IRAM Plateau de Bure Interferometer and the IRAM $30 \mathrm{~m}$ telescope on Pico Veleta. IRAM is supported by INSU/CNRS (France), MPG (Germany), and IGN (Spain).

$\star \star$ Data files (in FITS format) of the data presented in Figs. 6 and 7 are available at the CDS via anonymous ftp to

cdsarc.u-strasbg.fr $(130.79 .128 .5)$ or via

http://cdsweb.u-strasbg.fr/cgi-bin/qcat?J/A+A/475/559 shell and ii) stationarity of the outflow, i.e. the mass loss rate and the wind velocity are assumed to be constant in time.

There are serious doubts about the validity of these hypotheses. For instance, Mauron \& Huggins $(1999,2000)$ find evidence of variations in the mass loss rate of the carbon star IRC+10216 with timescales ranging from 80 years to a few hundreds of years. Kemper et al. (2003), when modeling CO rotational transitions from $J=2-1$ to $J=7-6$ in several sources, find indications of mass loss variations on the same timescales. Another spectacular example of episodic mass loss is revealed by the socalled detached shells observed in $\mathrm{CO}$ around some carbon-rich sources, notably TT Cyg, whose shell has been fully mapped with the Plateau de Bure interferometer (PdBI) (Olofsson et al. 2000). These shells are formed over a time span of about $1000 \mathrm{yr}$ and are thought to be triggered by thermal pulses (Olofsson et al. 1996, 2000; Schröder et al. 1999; Mattsson et al. 2007). Recently, Knapp et al. (1998) found several sources with composite CO rotational line profiles, i.e. a narrow component superimposed on a broader one, while both components are centered on the same LSR velocity.

Three different scenarios have been proposed to explain this kind of line profile:

i) Knapp et al. (1998) interpret them as due to 2 successive winds of different ages with different expansion velocities and corresponding to different mass loss rates. In this interpretation, 
the narrow component (which typically indicates velocities $<5 \mathrm{~km} \mathrm{~s}^{-1}$ and low mass loss rates $<10^{-7} M_{\odot} \mathrm{yr}^{-1}$ ) traces a more recent episode of mass loss after some changes in the stellar properties have occurred. This scenario would also apply to the early stage in the formation of a detached shell.

ii) Kahane \& Jura (1996) interpret the composite profile of $\mathrm{X}$ Her as being produced by the combination of a very slow wind in spherical expansion and of a faster bipolar flow. Neri et al. (1998) have obtained interferometric data at the PdBI showing in some cases evidence of bipolarity, notably in the case of RS Cnc, which also displays composite CO line profiles.

iii) For other sources, such as BM Gem, Jura \& Kahane (1999) propose that the narrow profile is due to a long-lived reservoir of orbiting molecular gas in Keplerian rotation. The line profile of this source is particularly narrow $(F W H M \sim$ $1 \mathrm{~km} \mathrm{~s}^{-1}$ ).

Until now, no definite answer to the question of the origin of these peculiar profiles could be given, mainly due to the lack of appropriate high-sensitivity, high angular-resolution observations of the corresponding objects.

To investigate the mass loss process of AGB stars, we have developed a consistent theoretical description to model the dustforming outflows from molecular atmospheres excited by periodic stellar pulsations. These models closely reproduce the general features of typical circumstellar winds around AGB stars with expansion velocities in the range 10 to $30 \mathrm{~km} \mathrm{~s}^{-1}$ and mass loss rates up to $\sim 10^{-4} M_{\odot} \mathrm{yr}^{-1}$. Additionally, we found a second class of models which develop slow winds, $v_{\exp }<5 \mathrm{~km} \mathrm{~s}^{-1}$, with low mass loss rates, $\dot{M}<3 \times 10^{-7} M_{\odot} \mathrm{yr}^{-1}$ (Winters et al. 2000). A transition between the two types of models can be the result of small changes in the stellar parameters, lending support to the Knapp et al. (1998) interpretation of the composite line profiles and providing a physical mechanism to explain the formation of detached shells (Schröder et al. 1999). In particular, even the low velocity derived from the BM Gem narrow CO-line component would still be compatible with our low mass loss rate models of spherically expanding winds.

To test these models, we have obtained $\operatorname{CO}(J=2-1)$, $\mathrm{CO}(J=1-0)$, and $\operatorname{SiO}(v=1, J=2-1)$ spectra at the IRAM-30 $\mathrm{m}$ and at the SEST on sources selected for their weak near-infrared excess (EP Aqr, $\mathrm{L}^{2}$ Pup, etc.); the results are in good agreement with the predictions of our model calculations (Winters et al. 2002, 2003, henceforth called Paper I). Furthermore, independent observational evidence based on 11.7 and $17.9 \mu \mathrm{m}$ images obtained at the Keck I telescope on $\mathrm{L}^{2}$ Pup has been presented by Jura et al. (2002), that also supports our low mass loss rate models.

Presently, about a dozen objects are known to show composite CO profiles. Out of these, we selected EP Aqr, a nearby source $(d \sim 135 \mathrm{pc})$ that is particularly bright in $\mathrm{CO}$ and that shows composite $\mathrm{CO}$ rotational line profiles with very distinct components. EP Aqr is indeed the source with the most contrasted composite CO profiles. Also it is relatively close to the Sun, so that we expect to resolve its circumstellar structure. Here we present detailed, velocity-resolved maps of EP Aqr in ${ }^{12} \mathrm{CO}(J=2-1)$ and ${ }^{12} \mathrm{CO}(J=1-0)$ in order to check which of the 3 scenarios for the structure of the circumstellar shell is correct, if any.

\section{Presentation of the source}

EP Aqr is an M-type (M8III) semi-regular variable (SRb) with a period of 55 days. From Hipparcos, its distance is derived
Table 1. Lines observed in EP Aqr.

\begin{tabular}{|c|c|c|c|}
\hline Line & $\begin{array}{c}v_{\mathrm{lsr}} \\
{\left[\mathrm{km} \mathrm{s}^{-1}\right]}\end{array}$ & $\begin{array}{c}v_{\exp } \\
{\left[\mathrm{km} \mathrm{s}^{-1}\right]}\end{array}$ & Ref* $^{*}$ \\
\hline $\mathrm{SiO}(3-2)$ & -31.9 & 7.9 & (1) \\
\hline $\mathrm{SiO}(2-1)$ & -31.9 & 8.2 & (1) \\
\hline \multirow[t]{2}{*}{$\mathrm{CO}(3-2)$} & -33.7 & 1.5 & (2) \\
\hline & -33.0 & 10.8 & (2) \\
\hline \multirow[t]{4}{*}{$\mathrm{CO}(2-1)$} & -33.8 & 1.4 & (2) \\
\hline & -33.0 & 10.8 & (2) \\
\hline & -34.0 & 1.0 & (3) \\
\hline & -34.0 & 11.0 & (3) \\
\hline \multirow[t]{3}{*}{$\mathrm{CO}(1-0)$} & -34.0 & 1.0 & (3) \\
\hline & -34.0 & 11.0 & (3) \\
\hline & & $\begin{array}{c}F W H M \\
{\left[\mathrm{~km} \mathrm{~s}^{-1}\right]}\end{array}$ & \\
\hline \multirow[t]{3}{*}{$\overline{\mathrm{HI}}$} & -31.1 & 13.3 & (4) \\
\hline & -26.4 & 2.6 & (4) \\
\hline & -31.0 & 1.6 & (4) \\
\hline
\end{tabular}

* References. (1) González Delgado et al. (2003); (2) Knapp et al. (1998); (3) Paper I; (4) Le Bertre \& Gérard (2004).

to be $\sim 135$ pc. In Paper I we estimated its luminosity to be $L_{\star} \approx 4800 L_{\odot}$, and Dumm \& Schild (1998) give an effective temperature of $T_{\star}=3236 \mathrm{~K}$ and a mass of $M_{\star}=1.7 M_{\odot}$. Knapp et al. (1998) and Winters et al. (2003) obtained CO line spectra at the source position that show clear composite profiles. The full width of the narrow feature is around $2 \mathrm{~km} \mathrm{~s}^{-1}$ and $22 \mathrm{~km} \mathrm{~s}^{-1}$ for the broad feature (see also Sect. 4.1). The CO photo-dissociation radius is estimated to be located at $1-2 \times 10^{16} \mathrm{~cm}$; therefore, we expect a shell size around 10-20 arcsec.

EP Aqr has been detected in H I at $21 \mathrm{~cm}$ with the Nançay Radiotelescope (Le Bertre \& Gérard 2004). The H I profile of EP Aqr is complex, with a broad and a narrow component centered at $v_{\mathrm{lsr}} \sim-31 \mathrm{~km} \mathrm{~s}^{-1}$ and a prominent emission feature at $v_{\text {lsr }} \sim-26 \mathrm{~km} \mathrm{~s}^{-1}$, which is almost absent in the CO profiles. The $\mathrm{HI}$ emission is extended ( $\sim 1 \mathrm{pc})$ and shows a line profile that varies radially and azimuthally. Using the Very Large Array (VLA), Matthews \& Reid (2007) have detected arcminute-scale $\mathrm{HI}$ emission features at velocities consistent with the H I emission profile reported by Le Bertre \& Gérard (2004). However, they cannot unambiguously conclude on the relation between these clumps and EP Aqr. EP Aqr has also been detected in the $\mathrm{SiO}$ thermal emission lines $(J=2-1$ and $J=3-2)$ (González Delgado et al. 2003). The spectral profiles show only one component centered at $-32 \mathrm{~km} \mathrm{~s}^{-1}$. Interestingly the central velocities of these different lines differ by $1-2 \mathrm{~km} \mathrm{~s}^{-1}$, with $v_{\mathrm{lsr}} \sim$ -33.0 to -34.0 for $\mathrm{CO},-32.0$ for $\mathrm{SiO}$, and -31.0 for $\mathrm{H} \mathrm{I}$ (Table 1 ). Surprisingly, the narrow $\mathrm{CO}$ and $\mathrm{HI}$ features basically do not overlap. As we expect the large-scale structures evolving from the structure of the circumstellar shell close to the central stars, we aimed for high-spatial resolution maps in $\mathrm{CO}$ with a spectral resolution corresponding to about $0.1 \mathrm{~km} \mathrm{~s}^{-1}$ in order to separate the different kinematic components.

Nakashima (2006) has obtained a $\mathrm{CO}(1-0)$ map with the BIMA interferometer. The spatial resolution is $\sim 4^{\prime \prime}$ and the velocity resolution $1 \mathrm{~km} \mathrm{~s}^{-1}$. The source was resolved, but no clear conclusion could be drawn regarding any separate kinematic wind components.

\section{Observations}

EP Aqr was observed with the Plateau de Bure interferometer in BCD configuration, first on Sep. 16, 2003 (4.9h with 5 antennas 
in D-configuration, $2 \mathrm{~mm}$ precipitable water vapor (pwv)), then on Dec. 8, 2003 (6.3 h with 6 antennas in C-configuration, pwv $\sim 1.5 \mathrm{~mm}$ ), and finally on Jan. 3, 2004 (6.9 h with 6 antennas in B-configuration, pwv $\sim 1.5 \mathrm{~mm}$ ). In order to obtain a sufficient spectral resolution, we used a correlator unit with a bandwidth of $20 \mathrm{MHz}$ and a channel spacing of $39 \mathrm{kHz}$ centered on the ${ }^{12} \mathrm{CO}(1-0)$ line and a unit with $40 \mathrm{MHz}$ bandwidth and $78 \mathrm{kHz}$ channel spacing centered on the ${ }^{12} \mathrm{CO}(2-1)$ line. This gives a velocity resolution for both lines of about $0.1 \mathrm{~km} \mathrm{~s}^{-1}$. The adjacent continuum in both bands was covered by two $320 \mathrm{MHz}$-wide units in each frequency band. The $3 \mathrm{~mm}$ receiver was tuned single sideband with the line placed in the upper sideband, whereas the $1 \mathrm{~mm}$ receiver was tuned double sideband, and the line was placed in the lower sideband. Typical SSB system temperatures were around $400 \mathrm{~K}$ at $115 \mathrm{GHz}$ and around $350 \mathrm{~K}$ at $230 \mathrm{GHz}$. The integrations on EP Aqr were interleaved every 20 min with short integrations ( $135 \mathrm{~s}$ ) on two nearby phase and amplitude calibrators (the quasars 2223-052 and 2145+067). Pointing and focus of the antennas were checked and, if necessary re-adjusted, every $\sim 65 \mathrm{~min}$. Subsequent data reduction was done with the GILDAS $^{1}$ software package (Pety 2005). The receiver bandpass was calibrated on the quasars 3C 454.3 (Jan. 2004) and 0420014 (Sep. and Dec. 2003), and the flux calibration was based on MWC 349. Only data with phase noise better than 40 deg at $115 \mathrm{GHz}$ were retained in the final $u v$-tables. The resulting useful on-source integration time with the PdBI is equivalent to $10 \mathrm{~h}$ with the six $15 \mathrm{~m}$ antennas.

Inspection of the resulting spectra and comparison with ${ }^{12} \mathrm{CO}(2-1)$ and (1-0) single-dish observations (Paper I) revealed that at least a factor two of the flux is lost from the interferometric observation due to the missing short spacing information. We therefore additionally obtained a spectral line on-thefly $\left(\right.$ OTF) map of EP Aqr in a $100^{\prime \prime} \times 100^{\prime \prime}$ field with the IRAM $30 \mathrm{~m}$ telescope. These observations were performed on May 68, 2004 under stable atmospheric conditions and with a pwv column of about $1.5 \mathrm{~mm}$. Each of our OTF maps consists of 21 lines spaced by $5^{\prime \prime}$ perpendicular to the scanning direction, and each line contains 25 dumps corresponding to a 4 " spacing on the sky in the scanning direction, fully sampling the beam of the $30 \mathrm{~m}$ telescope at $230 \mathrm{GHz}$. After each three such lines, a cold load calibration and a position switch with $400^{\prime \prime}$ offsets were performed in order to subtract the sky background. In total, we obtained 18 coverages of the $100^{\prime \prime} \times 100^{\prime \prime}$ field, with alternating scanning direction oriented perpendicular on the sky. We simultaneously used the $\mathrm{A} 100$ and $\mathrm{B} 100$ receivers at $2.6 \mathrm{~mm}$ and the A230 and B230 receivers at $1.3 \mathrm{~mm}$, and the data of both receivers in each band were averaged to increase the $S / N$ ratio. As backend we used the VESPA (VErsatile SPectrometer Assembly) autocorrelator with a spectral configuration and a delay apodization matching that of the PdBI observations. Linear baselines were subtracted from the spectra, and the data from all coverages in each band were merged to produce the final data cubes. Subsequently, the short spacing information was added to the $u v$-tables produced from the interferometric data. The combined observations cover a range of projected baselines from 0 to $\sim 330 \mathrm{~m}$; the final $u v$-coverage is shown in Fig. 1 . At $2.6 \mathrm{~mm}$ this coverage leads to a synthesized beam of $33^{\prime \prime} .53 \times 11^{\prime \prime} .84$ at a position angle $\mathrm{PA}=28^{\circ}$, and at $1.3 \mathrm{~mm}$ the synthesized beam is $1^{\prime \prime} 67 \times 00^{\prime} .94$ at $\mathrm{PA}=29^{\circ}$. The rms noise in the resulting maps is $42 \mathrm{mJy}$ per synthesized beam at $3 \mathrm{~mm}$ in $0.1 \mathrm{~km} \mathrm{~s}^{-1}$ spectral channels, and at $1 \mathrm{~mm}$ we reach a noise level of $51 \mathrm{mJy}$ per synthesized beam and $0.1 \mathrm{~km} \mathrm{~s}^{-1}$ channel.

\footnotetext{
${ }^{1}$ http://www.iram.fr/IRAMFR/GILDAS
}

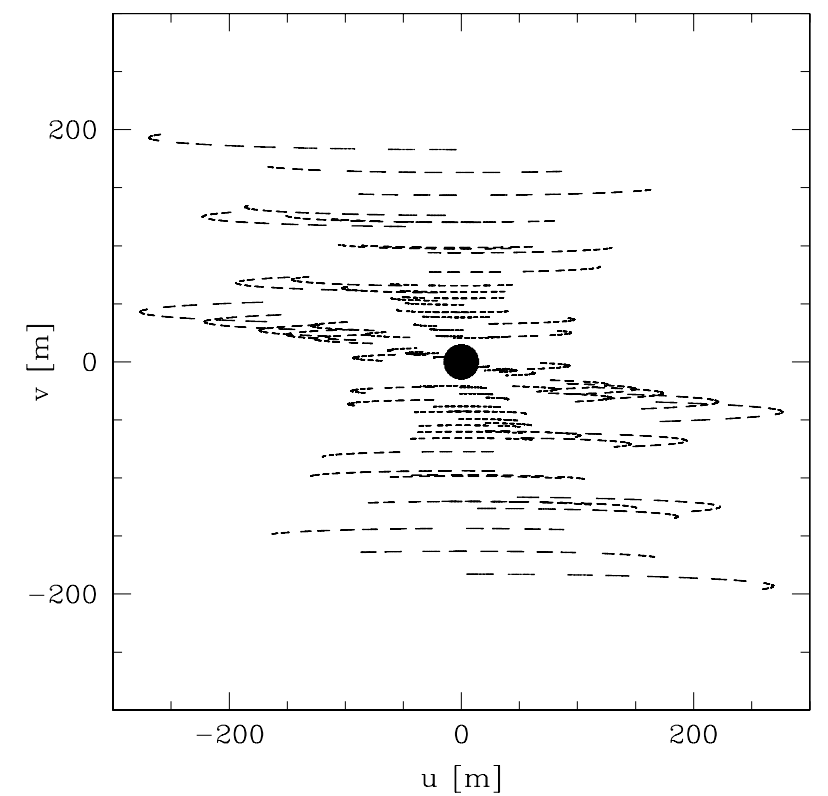

Fig. 1. $u v$-coverage of the combined PdBI and $30 \mathrm{~m}$ data.

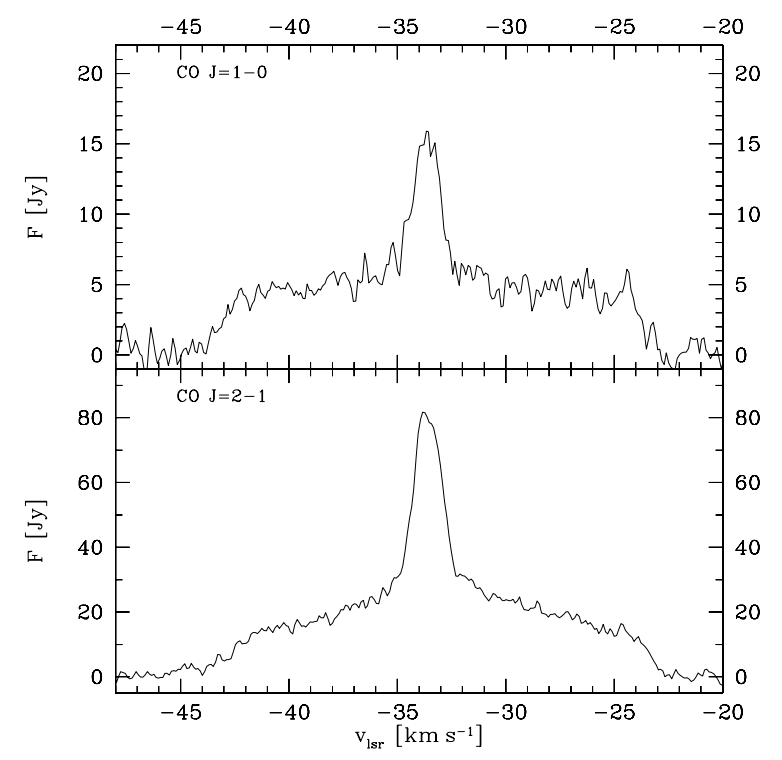

Fig. 2. ${ }^{12} \mathrm{CO}(J=1-0)$ (top) and (2-1) (bottom) line profiles of EP Aqr integrated over a $25^{\prime \prime} \times 25^{\prime \prime}$ field centered on the source position. The spectral resolution is $0.1 \mathrm{~km} \mathrm{~s}^{-1}$, and the components are centered on the stellar velocity $v_{\mathrm{lsr}}=-34 \mathrm{~km} \mathrm{~s}^{-1}$.

\section{Results}

\subsection{Spectra}

In Fig. 2 the map-integrated profiles of the ${ }^{12} \mathrm{CO}(1-0)$ and $(2-1)$ lines are displayed, both clearly showing a narrow component superimposed on a much broader one. Interpreting this composite profile as due to two wind components of different velocity, the narrow component would indicate an outflow-velocity of $v_{\exp } \sim 1 \mathrm{~km} \mathrm{~s}^{-1}$ and the broad component a velocity of $v_{\exp } \sim 11 \mathrm{~km} \mathrm{~s}^{-1}$. The missing flux in the interferometric maps compared with the single-dish observations from Paper I already indicated that the source is extended. Comparing now the flux integrated over a $25^{\prime \prime} \times 25^{\prime \prime}$ field with the flux recovered in the $11^{\prime \prime}$ and $22^{\prime \prime}(F W H M) 30 \mathrm{~m}$ beams at $230 \mathrm{GHz}$ and $115 \mathrm{GHz}$, respectively, we find a difference by factors of 1.6 and 1.8 for the 


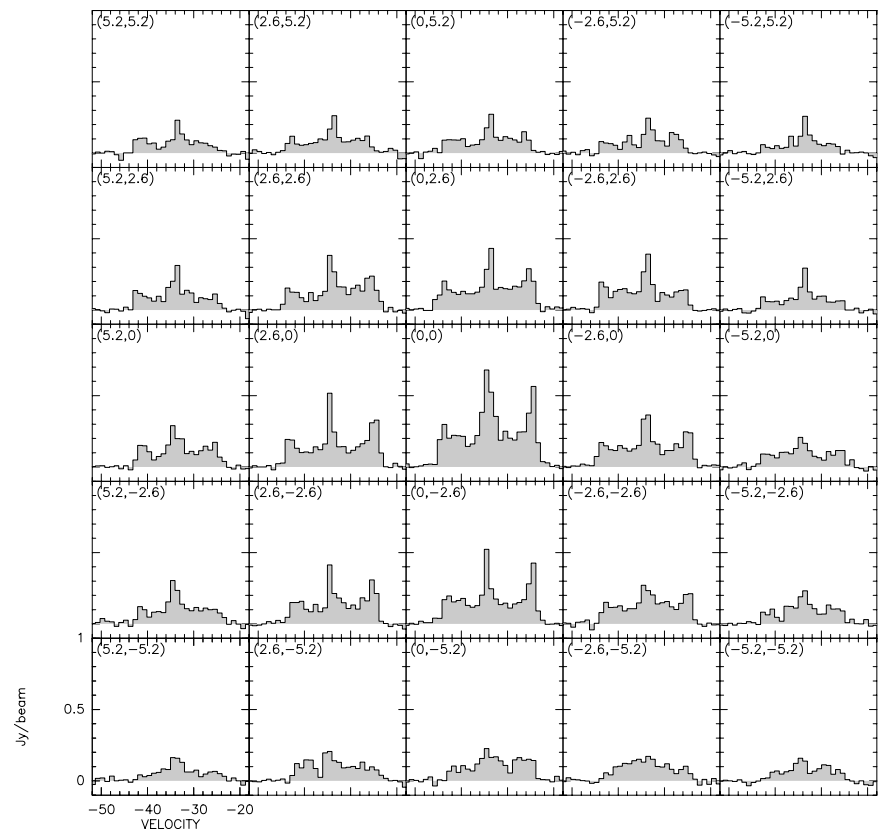

Fig. 3. Map of ${ }^{12} \mathrm{CO}(J=1-0)$ spectra in a $10^{\prime \prime}$ by $10^{\prime \prime}$ field centered on the source and spaced by $2.8^{\prime \prime}$ (the offset positions with respect to the phase center are given in the upper left corner of each diagram). The spectral resolution is $1 \mathrm{~km} \mathrm{~s}^{-1}$.

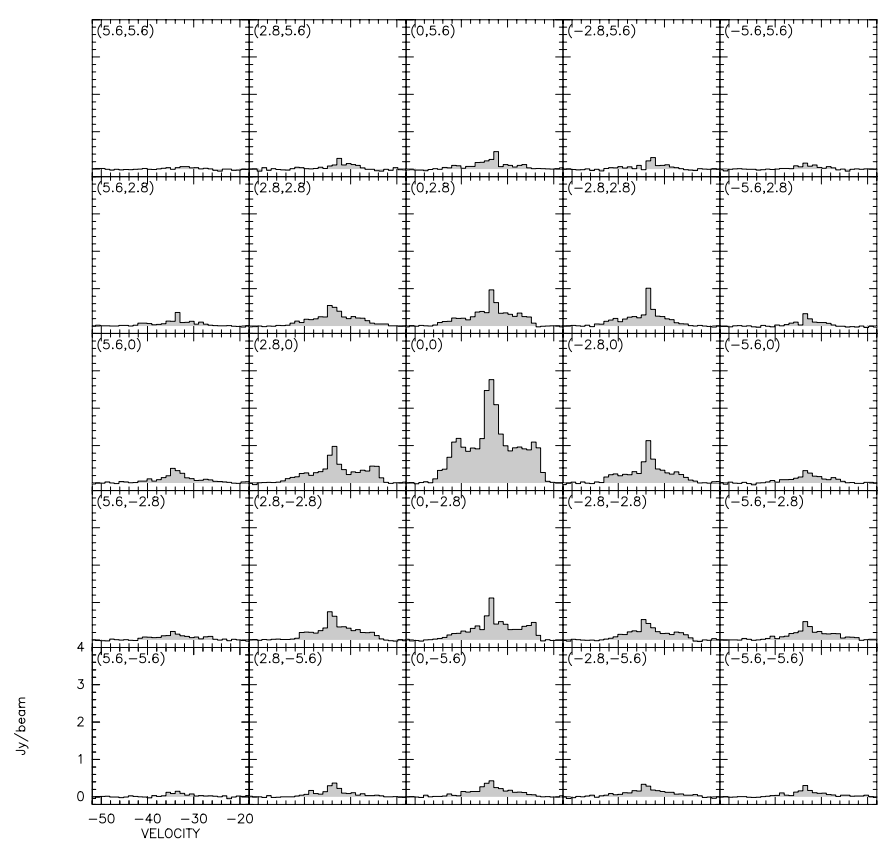

Fig. 4. Map of ${ }^{12} \mathrm{CO}(J=2-1)$ spectra in a $10^{\prime \prime}$ by $10^{\prime \prime}$ field centered on the source and spaced by $2.8^{\prime \prime}$ (the offset positions with respect to the phase center are given in the upper left corner of each diagram). The spectral resolution is $1 \mathrm{~km} \mathrm{~s}^{-1}$.

narrow and broad components at $1.3 \mathrm{~mm}$ and by a factor of 1.5 at $2.6 \mathrm{~mm}$ for both components, indicating that the source is already (partially) resolved in the $30 \mathrm{~m}$ beam at both frequencies.

The spatial variation of the ${ }^{12} \mathrm{CO}(1-0)$ and $(2-1)$ profiles is displayed in Figs. 3 and 4, respectively showing an overall roughly spherically-symmetric distribution of the molecular gas. From both transitions, there is evidence of low-velocity material in the outer part of the $\mathrm{CO}$ shell, whereas the high-velocity material appears to be concentrated closer to the star. In particular the "high-velocity" component seen around $v_{\mathrm{lsr}} \sim-24 \mathrm{~km} \mathrm{~s}^{-1}$ in

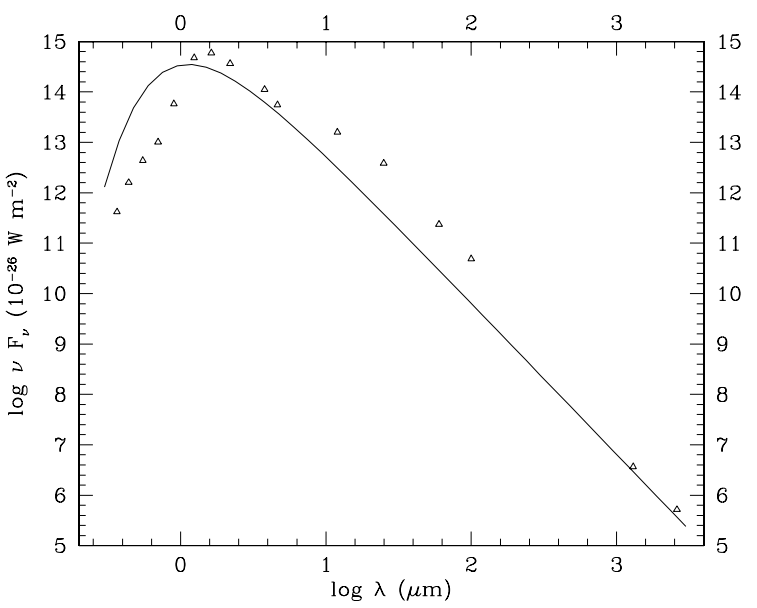

Fig. 5. Spectral energy distribution of EP Aqr. The solid line indicates the black body spectrum described in Sect. 4.2. UBVRI data: Moreno \& Carrasco (1986), JHKLM: Fouqué et al. (1992), IRAS: Beichman et al. (1988), mm data: this work.

the $\mathrm{CO}(1-0)$ line appears to arise from the inner $\pm 5^{\prime \prime}$ around the central position (see Fig. 3) and is slightly offset to the SE with respect to the map center.

\subsection{Continuum maps}

An unresolved continuum source is detected in both bands with a flux of $16 \mathrm{mJy}$ at $1.3 \mathrm{~mm}$ (rms noise $0.7 \mathrm{mJy}$ ) and $4.5 \mathrm{mJy}$ at $2.6 \mathrm{~mm}$ (rms noise $0.4 \mathrm{mJy}$ ), respectively, centered on the nominal position of EP Aqr (RA $21^{\mathrm{h}} 46^{\mathrm{m}} 31^{\mathrm{s}} .85$, Dec $-02^{\circ} 12^{\prime} 45^{\prime \prime} .92$ (2000.0)). Adopting a stellar temperature of $3236 \mathrm{~K}$ and a stellar radius of $166 R_{\odot}$ as given by Dumm \& Schild (1998), and assuming the HIPPARCOS distance of $135 \mathrm{pc}$, the black body law results in fluxes of $13 \mathrm{mJy}$ at $1.3 \mathrm{~mm}$ and $3 \mathrm{mJy}$ at $2.6 \mathrm{~mm}$. The spectral energy distribution of EP Aqr is shown in Fig. 5, together with this black body curve. The measured continuum fluxes in the $\mathrm{mm}$ range therefore seem compatible with the emission from the stellar photosphere. On the other hand, the infrared flux of EP Aqr is clearly dominated by dust: at $100 \mu \mathrm{m}$ IRAS measured a flux of $16 \mathrm{Jy}$, whereas the stellar black body would only yield $2.1 \mathrm{Jy}$.

\subsection{Channel maps}

In Figs. 6 and 7 we present channel maps in the ${ }^{12} \mathrm{CO}(1-0)$ and ${ }^{12} \mathrm{CO}(2-1)$ lines, respectively, that cover the central $35^{\prime \prime}$ and $25^{\prime \prime}$ around the stellar position. The channels are smoothed to a resolution of $1 \mathrm{~km} \mathrm{~s}^{-1}$. As already indicated by the spectral maps in Figs. 3 and 4, the circumstellar CO shell of EP Aqr appears to be roughly spherically symmetric also in the channel maps, though with a somewhat clumpy overall structure. Near the central channels, a slight non-sphericity at the lower contour levels seems to be present, however, with a somewhat larger extension in the southwest direction. This will be further addressed in Sect. 4.5.

Gaussian fits to the data cubes were performed in the $u v$-plane, and the results are displayed in Figs. 8 and 9. The central position of the Gaussian components was fixed at the phase center, i.e. at the nominal position of EP Aqr. Fitting also the source position does not improve the results. Elliptical Gaussian fits were tried, but no significant deviation from circular symmetry was found. In the following we will therefore assume circular symmetry of the source. 


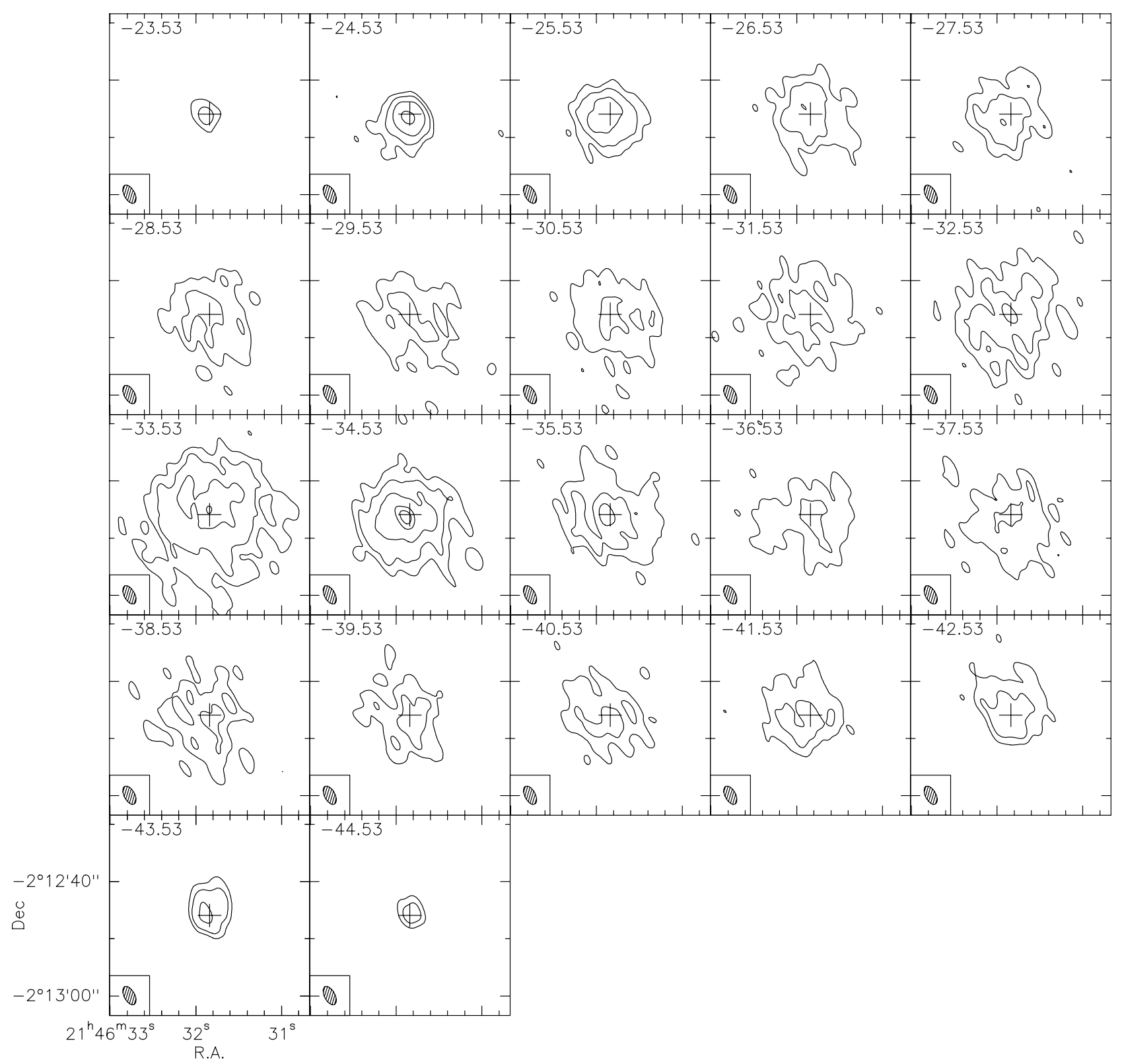

Fig. 6. Channel maps in the ${ }^{12} \mathrm{CO}(J=1-0)$ line (smoothed to a width of $\left.1 \mathrm{~km} \mathrm{~s}^{-1}\right)$. Contours are plotted at 5, 10, 20, 30, 40 $\sigma(1 \sigma=13 \mathrm{mJy} / \mathrm{beam})$. The maps are centered on the nominal position of EP Aqr: RA $21^{\mathrm{h}} 46^{\mathrm{m}} 31^{\mathrm{s}} .85$, Dec $-02^{\circ} 12^{\prime} 45^{\prime \prime} \cdot 92$ (2000.0). The synthesized beam is indicated in the lower left corner.

\section{$\operatorname{CO}(1-0):$}

The ${ }^{12} \mathrm{CO}(1-0)$ channel maps are well-fitted by one circular Gaussian component, a more complex decomposition of the source does not yield any qualitative improvement of the fit. We find a $(F W H M)$ diameter of the high outflow-velocity component (i.e. $v_{\exp } \sim 11 \mathrm{~km} \mathrm{~s}^{-1}$ ) of about $16^{\prime \prime}$. A more compact structure with an FWHM of $10^{\prime \prime}$ is found to reproduce the channel maps around $-34.9 \mathrm{~km} \mathrm{~s}^{-1}$. The velocity of this component is shifted by about $1 \mathrm{~km} \mathrm{~s}^{-1}$ with respect to the narrow emission peak at $-34 \mathrm{~km} \mathrm{~s}^{-1}$. The feature at $-24 \mathrm{~km} \mathrm{~s}^{-1}$ (see Fig. 3) can also be seen in the Gaussian fit. Its position is slightly offset toward the SE with respect to the phase center (Fig. 3), and it appears to be more compact, with its diameter of about $7^{\prime \prime}$ (see upper panel in Fig. 8), than the region emitting the narrow line component at $-34 \mathrm{~km} \mathrm{~s}^{-1}$. The entire $\mathrm{CO}(1-0)$ emitting region appears to be more extended than the $\mathrm{CO}(2-1)$ emitting region (see below) by a factor of about 2 .

\section{$\operatorname{CO}(2-1):$}

The ${ }^{12} \mathrm{CO}(2-1)$ emission maps are well-fitted by one circular Gaussian component as well. This component is characterized by an $F W H M$ of about $8^{\prime \prime}$, covering the full velocity range of the broad line profile. The narrow component of the line profile is thus not reflected by a geometrically different emission region in this fit. In fact, the source extension as a function of velocity as provided by this Gaussian fit component is exactly what we would expect from a spherical envelope with outer radius $R_{\mathrm{env}}$, expanding at constant velocity $v_{\text {exp }}$ :

$R(v)=R_{\mathrm{env}} \sqrt{\left(1-\left(\frac{v-v_{\mathrm{lsr}}}{v_{\mathrm{exp}}}\right)^{2}\right)}$

Fitting Eq. (1) to the FWHM plotted in Fig. 9 results in an envelope extension of $R_{\text {env }}=8.1^{\prime \prime}$, an expansion velocity $v_{\exp }=$ $11.2 \mathrm{~km} \mathrm{~s}^{-1}$, and a stellar velocity $v_{\mathrm{lsr}}=-33.6 \mathrm{~km} \mathrm{~s}^{-1}$. Also in 


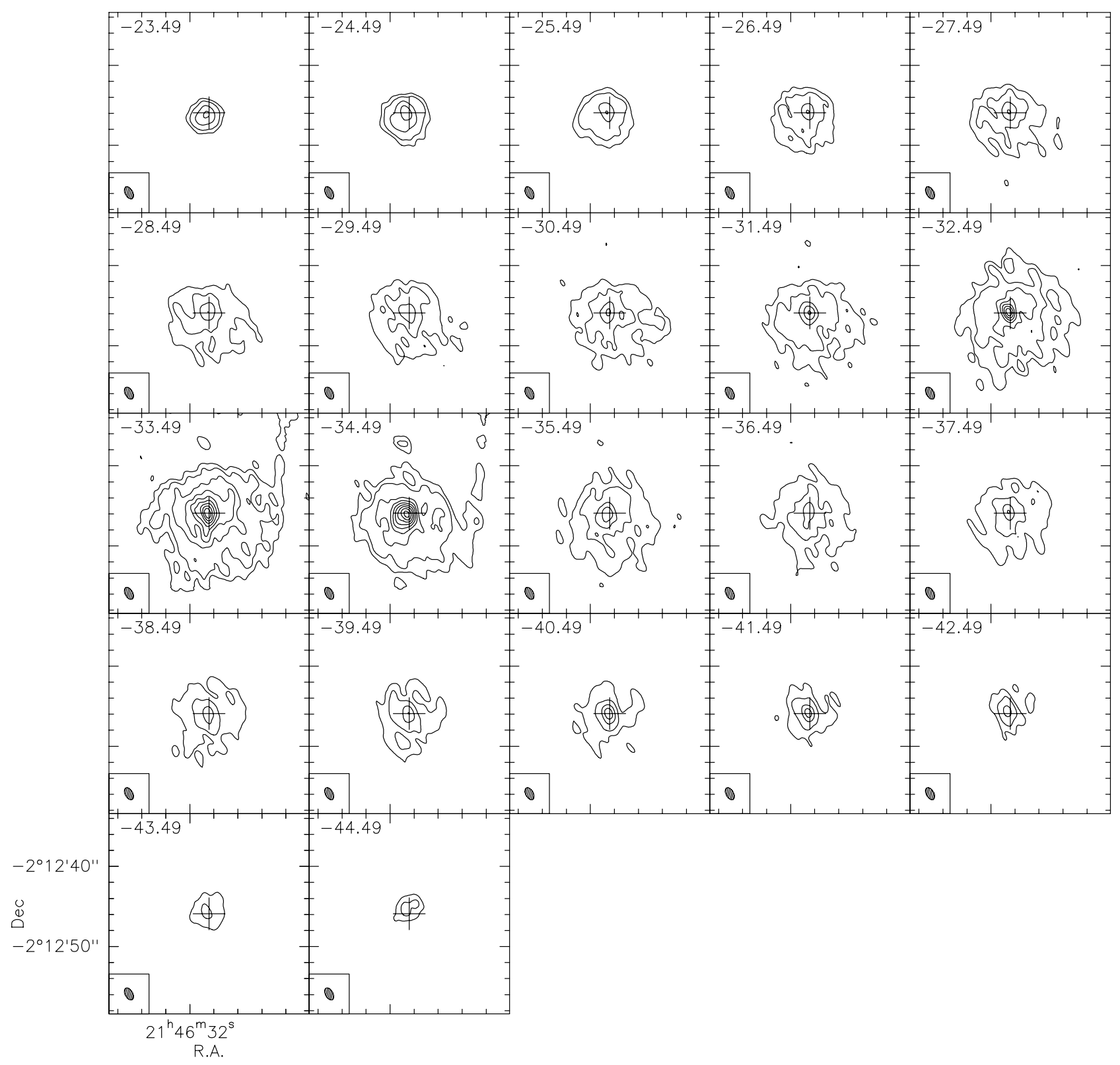

Fig. 7. Channel maps in the ${ }^{12} \mathrm{CO}(J=2-1)$ line (smoothed to a width of $1 \mathrm{~km} \mathrm{~s}^{-1}$ ). The first contour is plotted at $10 \sigma$, and the following contours start at $20 \sigma$ and are plotted in $20 \sigma$ steps $(1 \sigma=16 \mathrm{mJy} / \mathrm{beam})$. The maps are centered on the nominal position of EP Aqr: RA $21^{\mathrm{h}} 46^{\mathrm{m}} 31^{\mathrm{s}} .85$, Dec $-02^{\circ} 12^{\prime} 45^{\prime \prime} 92$ (2000.0). The synthesized beam is indicated in the lower left corner.

the higher spatially resolved (2-1) line, we therefore do not find a spatially different region that might be contributing to the narrow component of the composite line profile.

This absence of a spatial separation between the different kinematic components is corroborated by maps in the integrated profiles of both the (1-0) and (2-1) lines. In Figs. 10 and 11, we present contour maps integrated over the red and blue wings of the broad line component and integrated over the narrow component for both transitions. In both lines, there are no significant differences, whether in the location, shape, or the spatial extension, among the three different velocity regimes. A small non-sphericity at the lower contour levels, indicating a larger extension of the source toward the southwest, is apparent also in the integrated maps, notably close to the central velocities. This non-sphericity is more pronounced in the $\mathrm{CO}(1-0)$ line (see Fig. 10).

\subsection{Azimuth averages}

To better visualize the radial brightness distribution, azimuthal averages of the central channel maps in both lines (see Figs. 6 and 7 at $\sim-33.5 \mathrm{~km} \mathrm{~s}^{-1}$ ) were calculated and are displayed in Figs. 12 and 13. In the $\mathrm{CO}(1-0)$ central channel, the EP Aqr circumstellar shell shows a rather smooth average-brightness profile with a shallow shoulder at a projected distance of about $5^{\prime \prime}$.

In contrast, the $\mathrm{CO}(2-1)$ azimuthally-averaged brightness profile exhibits at least two pronounced intermediate maxima at projected distances of $3.5^{\prime \prime}$ and $7.5^{\prime \prime}$ from the phase center. We recall that the spatial resolution in the (2-1) line is a factor two higher than for the (1-0) line. These brightness maxima can be interpreted in terms of density variations in the circumstellar shell that might point to mass loss variations in the wind of EP Aqr (see Sect. 5.2). 


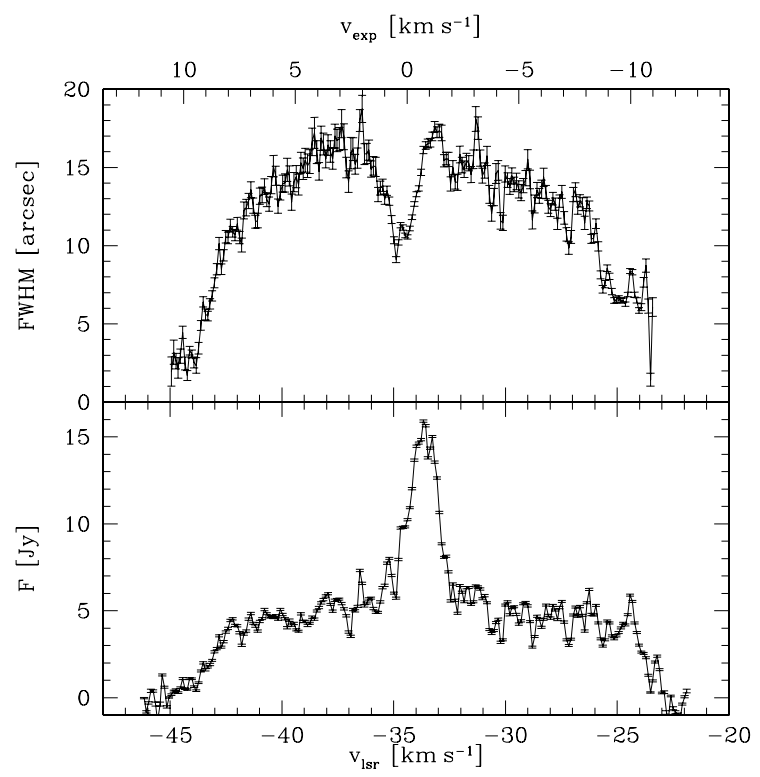

Fig. 8. Single-component circular Gaussian fit to the $\operatorname{CO}(J=1-0)$ channel maps. Upper panel: $F W H M$ as a function of velocity; lower panel: fitted flux. The spectral resolution is $0.1 \mathrm{~km} \mathrm{~s}^{-1}$.

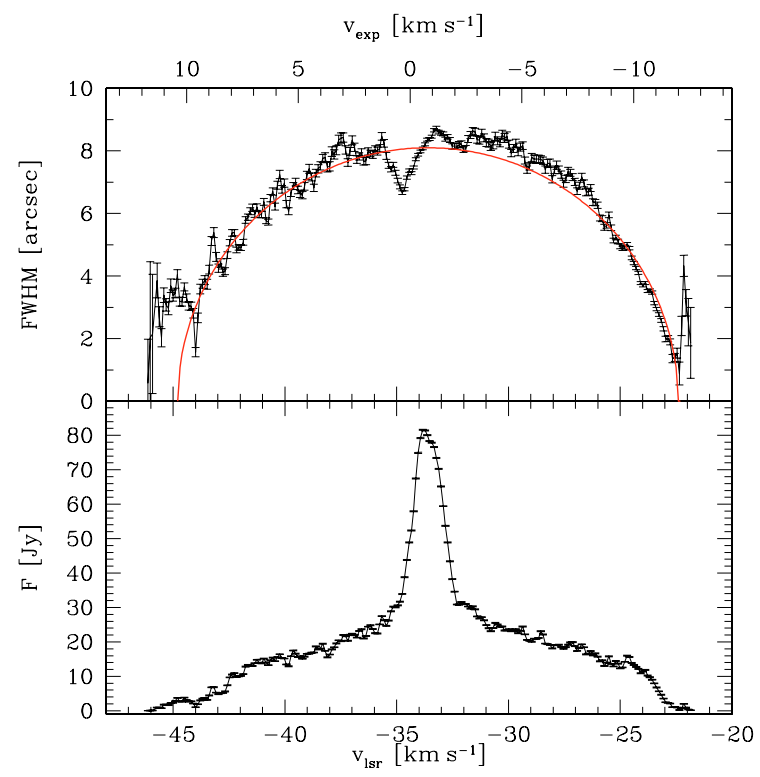

Fig. 9. Single-component circular Gaussian fit to the $\operatorname{CO}(J=2-1)$ channel maps. Upper panel: $F W H M$ as a function of velocity, together with the fit resulting from Eq. (1); lower panel: Gaussian fitted flux. The spectral resolution is $0.1 \mathrm{~km} \mathrm{~s}^{-1}$.

\subsection{Position velocity diagram in the $\mathrm{CO}(1-0)$ line center}

The slightly larger extension of the maps toward the southwest direction visible in the central channels displayed in Figs. 6, 7,10 , and 11 , as well as the dip seen around $-35 \mathrm{~km} \mathrm{~s}^{-1}$ in the circular Gauss fits to the source extension (upper panels of Figs. 8 and 9) points to the presence of some non-spherical structure at low outflow-velocities. In Fig. 14 a position-velocity diagram is depicted for the central velocity range between -31 and $-37 \mathrm{~km} \mathrm{~s}^{-1}$ in the $\mathrm{CO}(1-0)$ line. Offset positions are shown along a cut in southeast northwest direction, i.e. at a position angle of $-45^{\circ}$. In Fig. 14, a small velocity gradient across the central position can be seen, which peaks at $-34.5 \mathrm{~km} \mathrm{~s}^{-1}$ at an offset position of $\sim+2^{\prime \prime}$ and at $-33.5 \mathrm{~km} \mathrm{~s}^{-1}$ at $\sim+2^{\prime \prime}$. Note that this

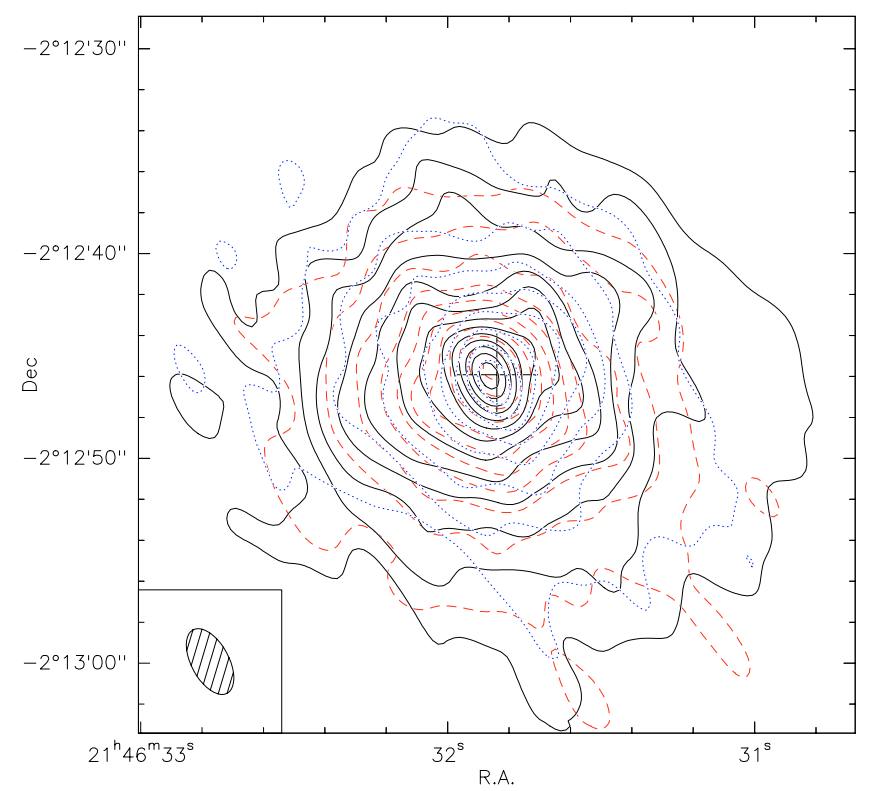

Fig. 10. Contour map in the ${ }^{12} \mathrm{CO}(J=1-0)$ line, integrated between: red (dashed): $-23 \mathrm{~km} \mathrm{~s}^{-1}$ and $-32 \mathrm{~km} \mathrm{~s}^{-1}$ (red wing of the broad line component), black (solid) $-32 \mathrm{~km} \mathrm{~s}^{-1}$ and $-36 \mathrm{~km} \mathrm{~s}^{-1}$ (narrow line component), and blue (dotted) $-36 \mathrm{~km} \mathrm{~s}^{-1}$ and $-45 \mathrm{~km} \mathrm{~s}^{-1}$ (blue wing of the broad line component). Contours are plotted in $5 \sigma$ steps, starting at $5 \sigma\left(1 \sigma=43.6 \mathrm{mJy} / \mathrm{beam} \mathrm{km} \mathrm{s}^{-1}\right.$ for the red and blue wings, and $28.8 \mathrm{mJy} /$ beam km s${ }^{-1}$ for the narrow component). The synthesized beam is indicated in the lower left corner.

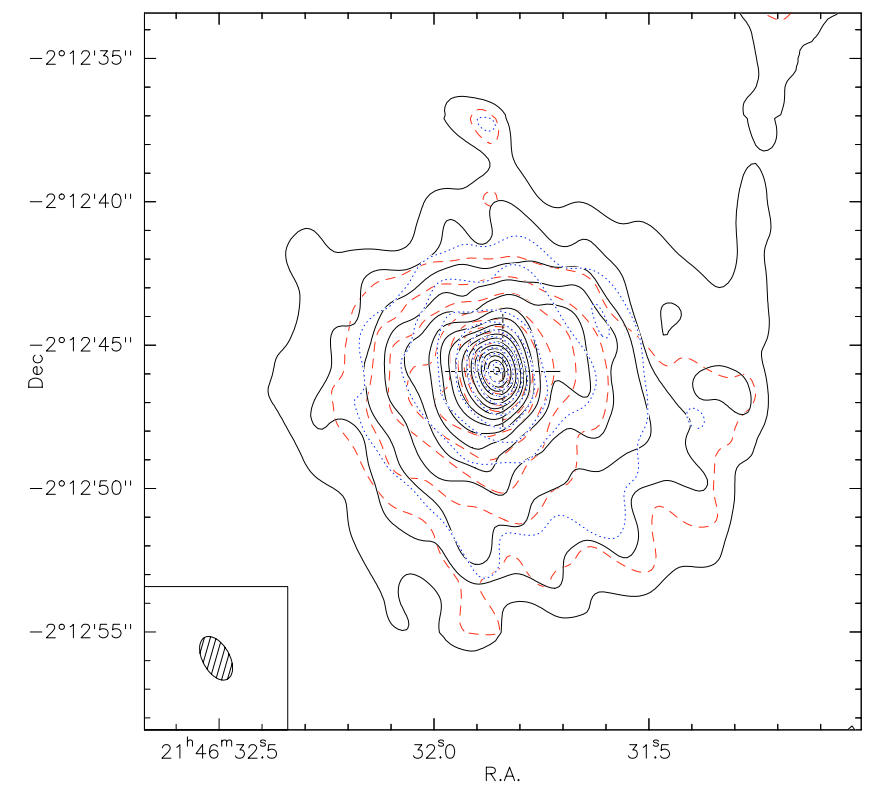

Fig. 11. Contour map in the ${ }^{12} \mathrm{CO}(J=2-1)$ line, integrated between: red (dashed): $-23 \mathrm{~km} \mathrm{~s}^{-1}$ and $-32 \mathrm{~km} \mathrm{~s}^{-1}$ (red wing of the broad line component), black (solid) $-32 \mathrm{~km} \mathrm{~s}^{-1}$ and $-36 \mathrm{~km} \mathrm{~s}^{-1}$ (narrow line component), and blue (dotted) $-36 \mathrm{~km} \mathrm{~s}^{-1}$ and $-45 \mathrm{~km} \mathrm{~s}^{-1}$ (blue wing of the broad line component). Contours are plotted in $15 \sigma$ steps, starting at $15 \sigma\left(1 \sigma=48.8 \mathrm{mJy} / \mathrm{beam} \mathrm{km} \mathrm{s}^{-1}\right.$ for the red and blue wing, and $32.3 \mathrm{mJy} / \mathrm{beam} \mathrm{km} \mathrm{s}{ }^{-1}$ for the narrow component). The synthesized beam is indicated in the lower left corner.

gradient appears in the southeast northwest direction, and does not seem to be directly related to the non-sphericity seen only toward the southwest. This velocity gradient can also be seen in Fig. 6, where the image at $-33.53 \mathrm{~km} \mathrm{~s}^{-1}$ is slightly shifted with respect to that at $-34.53 \mathrm{~km} \mathrm{~s}^{-1}$, but this position shift is 


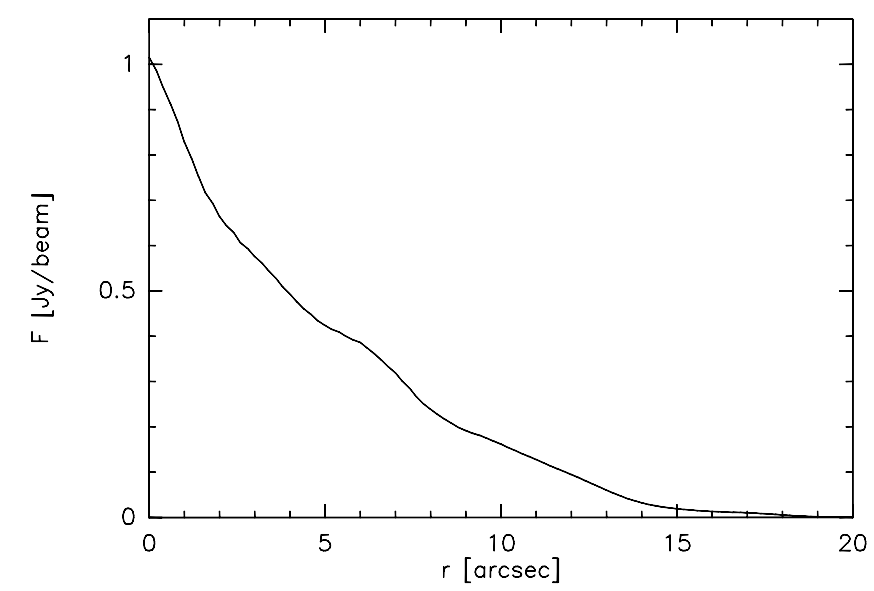

Fig. 12. Azimuthal average of the $\mathrm{CO}(J=1-0)$ brightness distribution in the central channel. The spectral resolution is $1 \mathrm{~km} \mathrm{~s}^{-1}$.

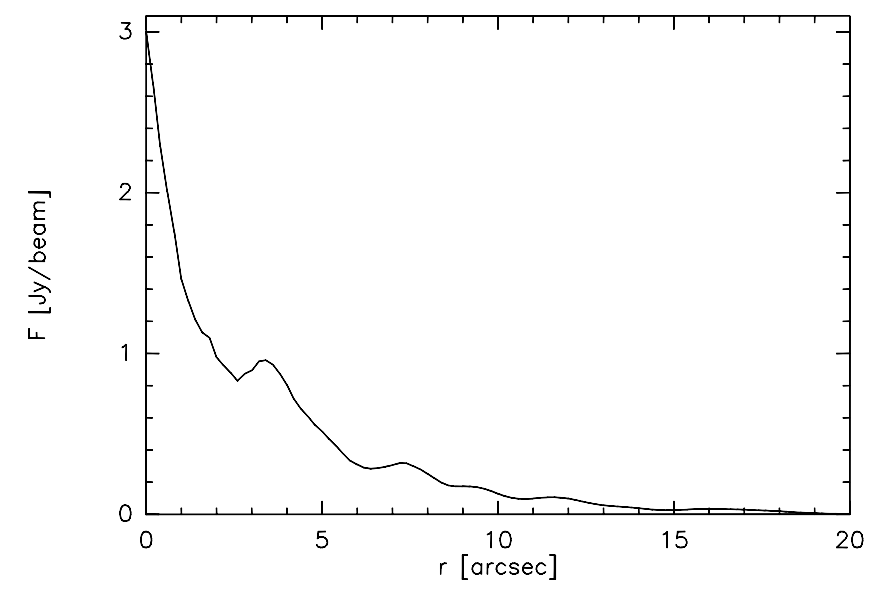

Fig. 13. Azimuthal average of the $\mathrm{CO}(J=2-1)$ brightness distribution in the central channel. The spectral resolution is $1 \mathrm{~km} \mathrm{~s}^{-1}$.

well within the beam size. We tried position-velocity diagrams in other directions, and also for the $\operatorname{CO}(2-1)$ line, but no discernible effect was found.

\section{Discussion}

\subsection{Monte-Carlo radiative transfer simulations}

To investigate the possible spatio-kinematic structure of the CO shell around EP Aqr in more detail, we performed MonteCarlo (MC) radiative transfer simulations assuming spherically symmetric configurations. For this purpose we utilized the 1D molecular line Monte-Carlo code of Hogerheijde \& van der Tak (2000) supplemented by the CO molecular data file from the LAMDA data base ${ }^{2}$, which is described in Schöier et al. (2005). Our aim is to reproduce as much as possible i) the observed $\mathrm{CO}(J=2-1)$ and (1-0) line profiles, ii) the azimuthallyaveraged brightness distributions, and iii) the maps of spectra. It turns out that combining this information puts strong constraints on the possible structure of the EP Aqr circumstellar shell.

We characterize the stellar photosphere by a blackbody with temperature $3236 \mathrm{~K}$ and radius $1.16 \times 10^{13} \mathrm{~cm}$. The kinetic gastemperature profile that determines the collision rates between $\mathrm{CO}$ and $\mathrm{H}_{2}$ is assumed to follow an $r^{-0.5}$ law out to a distance

\footnotetext{
${ }^{2}$ http://www.strw. leidenuniv.nl/ moldata/
}

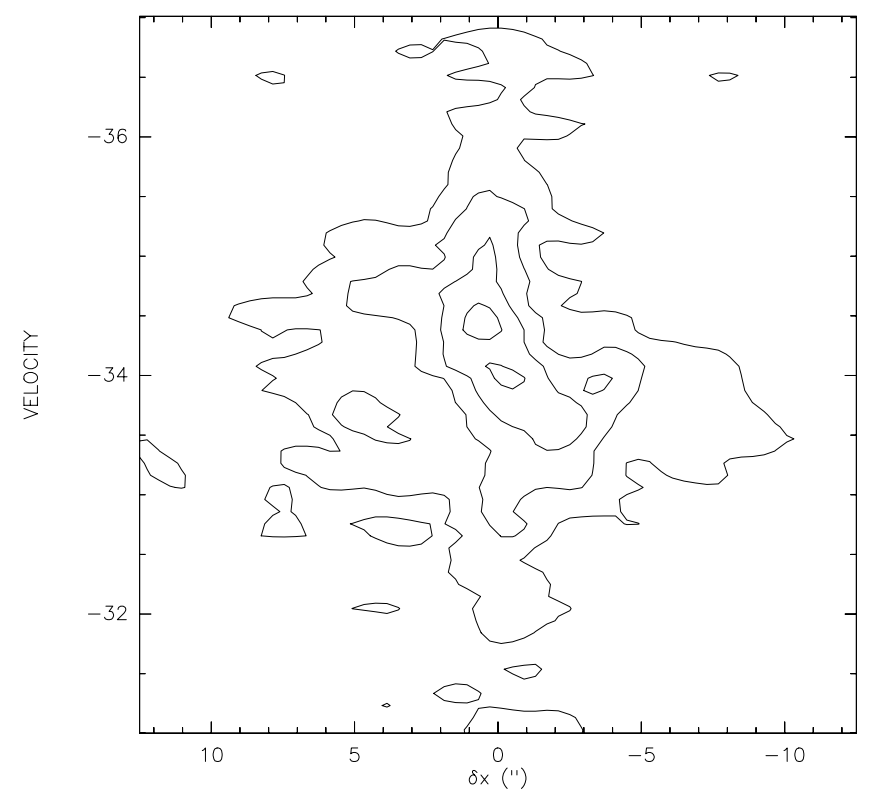

Fig. 14. Position-velocity diagram in the $\mathrm{CO}(1-0)$ line along a cut at $\mathrm{PA}$ $-45^{\circ}$. The spectral resolution is $0.1 \mathrm{~km} \mathrm{~s}^{-1}$. Contours are plotted in $5 \sigma$ steps, starting at $5 \sigma(1 \sigma=42 \mathrm{mJy} / \mathrm{beam})$.

of $10^{15} \mathrm{~cm}$ and an $1 / r$ law farther out. This is motivated by the results of, e.g., Jura et al. (1988) and Kastner (1992) (see also Teyssier et al. 2006). For the dust temperature distribution that influences the radiative transition rates, we assume an $r^{-0.5}$ law throughout the circumstellar envelope, i.e. we assume an optically thin radiative equilibrium temperature. The dust opacity is parameterized by the Ossenkopf \& Henning (1994) opacities for non-coagulated particles without ice mantles, and we assume a gas-to-dust mass ratio of 250 . The simulation domain is divided into a number of radial shells on a stepwise logarithmic grid. The CO model molecule contains the first 41 rotational levels in the vibrational and electronic ground state, coupled by the 40 allowed radiative transitions and by all possible 820 collisional transitions, taking into account collisions with para- $\mathrm{H}_{2}$ and ortho- $\mathrm{H}_{2}$ assumed to obey the equilibrium abundance ratio of $1 / 3$. In the simulations we assume a constant micro-turbulence velocity of $0.5 \mathrm{~km} \mathrm{~s}^{-1}$ (see Olofsson et al. 2002).

The final data cubes resulting from the Monte-Carlo simulations are converted into the GILDAS data format and can therefore be treated in exactly the same way as the observed data. To compare the calculated results directly with our observations, the simulated images were convolved with the actual synthesized beam of the combined interferometric and OTF observations, and we added the respective noise in the observed maps to the calculated data cubes.

It is obvious that a spherically symmetric model with a constant mass loss rate and a constant outflow-velocity in the region where the $\mathrm{CO}$ rotational lines are formed cannot reproduce the observed two-component line profiles.

Assuming two independent successive winds, in Paper I we fit the composite line profiles observed with the $30 \mathrm{~m}$ telescope by two independent parabola and derived mass loss rates and outflow-velocities of $\sim 5 \times 10^{-8} M_{\odot} \mathrm{yr}^{-1}$ and $1 \mathrm{~km} \mathrm{~s}^{-1}$ from the narrow component of the line profiles and $\sim 8 \times 10^{-7} M_{\odot} \mathrm{yr}^{-1}$ and $11 \mathrm{~km} \mathrm{~s}^{-1}$ from the broad component.

In fact, the profiles resulting from a $\mathrm{MC}$ calculation in a spherical 2-component wind model with the characteristics summarized in Table 2 reproduce the observed profiles reasonably 


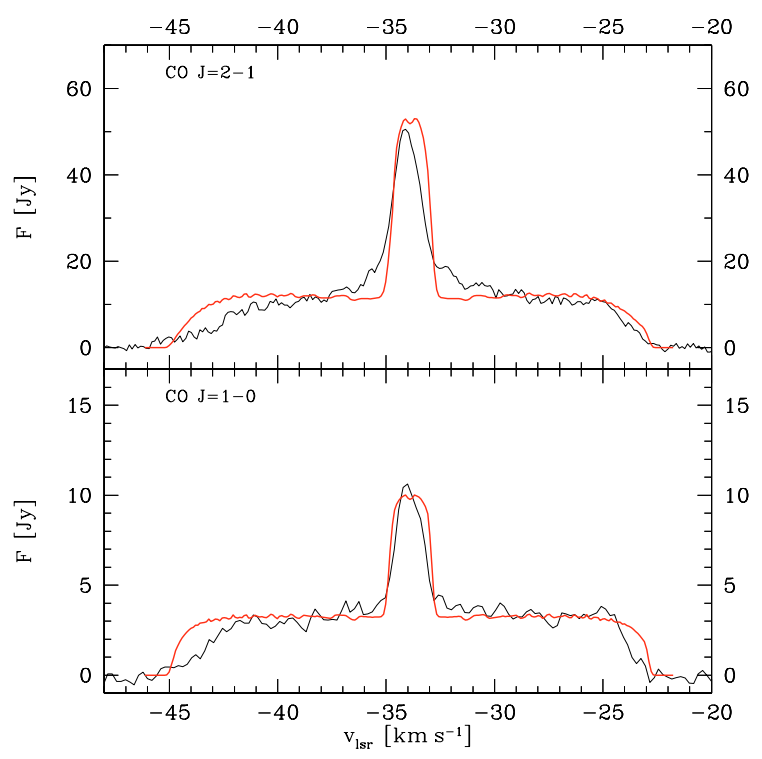

Fig. 15. ${ }^{12} \mathrm{CO}(J=2-1)$ (top) and (1-0) (bottom) line profiles of EP Aqr observed with the $30 \mathrm{~m}$ telescope (from Paper I). The spectral resolution is $0.1 \mathrm{~km} \mathrm{~s}^{-1}$. Overlaid are the profiles resulting from an MC simulation for a two-wind model (see Table 2) described in the text.

Table 2. Parameters of the wind model discussed in the text.

\begin{tabular}{c|ccccc}
\hline \hline & $\begin{array}{c}\dot{M} \\
M_{\odot} \mathrm{yr}^{-1}\end{array}$ & $\begin{array}{c}v_{\star} \\
{\left[\mathrm{km} \mathrm{s}^{-1}\right]}\end{array}$ & $\begin{array}{c}v_{\exp } \\
{\left[\mathrm{km} \mathrm{s}^{-1}\right]}\end{array}$ & $\begin{array}{c}r_{\text {in }} \\
{[\text { “"] }}\end{array}$ & $\begin{array}{c}r_{\text {out }} \\
{[\text { [“] }}\end{array}$ \\
\hline comp 1 & $2.0 \times 10^{-8}$ & 34.0 & 1.0 & 0.01 & 6.00 \\
comp 2 & $6.0 \times 10^{-7}$ & 34.0 & 11.0 & 6.00 & 10.0 \\
\hline
\end{tabular}

well. (See Fig. 15; the calculated profiles are convolved with the respective beam of the $30 \mathrm{~m}$ telescope and no micro-turbulent Doppler broadening was invoked in this line calculation.) The mass loss rates adopted for these two components are in reasonable agreement with those obtained in Paper I, and the outer radii of both wind components are compatible with the estimated photo-dissociation radii (see Knapp et al. 1998, Paper I).

A simple two-wind model is, however, capable of reproducing neither the structures seen in the azimuthally averaged brightness profiles (see Figs. 12 and 13) nor the shape of the spectra along the central (and other) lines of sight (see Figs. 3 and 4). Notably from the spectra mapped with the PdBI along single lines of sight, it is evident that dense material is contributing to the emission at intermediate velocities. In a spherical model, this could be contributed by projected velocities of a "fast" wind (i.e. $v_{\exp } \approx 10 \mathrm{~km} \mathrm{~s}^{-1}$ ). To avoid the occurrence of pronounced peaks in the spectra at the terminal velocity, the (fast) wind needs, however, to be unresolved by the interferometric observation, i.e. the outer radius of the fast wind needs to be smaller than about $1^{\prime \prime}$ in our case. A fast spherical wind would therefore need to be confined to the region very close to the star, corresponding to $2 \times 10^{15} \mathrm{~cm}$ or, equivalently, to a timescale of about $60 \mathrm{yr}$ for the outflow-velocity of $11 \mathrm{~km} \mathrm{~s}^{-1}$. Such a small extension of the massive wind component, however, contradicts both the relatively shallow increase in the azimuthally averaged brightness profile and the results of the Gaussian fits (see Figs. 8 and 9) that indicate an extension of the fast wind component on the order of $10^{\prime \prime}$. Moreover, such a compact component, due to beam dilution, would yield a much lower signal with the $30 \mathrm{~m}$ telescope than is actually observed.
We have tried other models with multiple successive winds that have different outflow-velocities. It is possible to improve the fits, but the exercise becomes somewhat unconstrained. Also, we were never able to get a satisfactory line profile at the center of the map. We suspect that some inhomogeneities within the outflows, without impacting on the general spherical structure of the circumstellar shell, may affect the radiation transport. Indeed, the contour maps (Figs. 6 and 7) provide some indications of clumpiness.

Another source of concern is that in our simulations $\mathrm{CO}$ is excited through collisions with molecular hydrogen. In fact, hydrogen might instead be mostly atomic in the circumstellar shell of EP Aqr, as the stellar effective temperature is $\sim 3200 \mathrm{~K}$. Glassgold \& Huggins (1983) have predicted that most hydrogen should be atomic in atmospheres and outflows of stars with a stellar effective temperature $\geq 2500 \mathrm{~K}$. The detection of an intense emission in the HI line at $21 \mathrm{~cm}$ toward EP Aqr by Le Bertre \& Gérard (2004) confirms the presence of atomic hydrogen in its circumstellar shell and more generally gives support to the Glassgold \& Huggins prediction.

\subsection{Interpretation}

We observe images that are virtually circular-symmetric and centered on the star's optical position, and spectra that are basically symmetric with respect to the stellar LSR velocity. The present data show very little evidence of non-sphericity in the outflow region that is probed by the CO (2-1) and (1-0) lines. This result does not support a bipolar model for EP Aqr such as the one proposed by Kahane \& Jura (1996) for X Her.

In our simulations, the sizes that we obtain for the $\mathrm{CO}$ emission agree with the photo-dissociation radii estimated by Knapp et al. (1998) and in Paper I. These simulations, although not entirely satisfactory, support their suggestion that the very narrow component in the CO spectra of EP Aqr represents a new phase of mass loss.

Such a change in the wind characteristics (outflow-velocity and mass loss rate) may be the result of small changes in the stellar parameters of a dust-forming, pulsating AGB star (Winters et al. 2000). In Paper I we presented observations of the $\mathrm{SiO}(v=$ $1, J=2-1$ ) maser line arising from EP Aqr (see their Fig. 11). This line is supposed to be formed close to the stellar photosphere at $\sim 1-2 R_{*}$; see, e.g., Greenhill et al. (1995); Boboltz et al. (1997), and Wittkowski et al. (2007) for the $43 \mathrm{GHz}$ maser line. The velocity indicated by the $86 \mathrm{GHz}$ maser features $\left(\sim 4 \mathrm{~km} \mathrm{~s}^{-1}\right.$ with respect to the stellar LSR velocity) is clearly in excess of the velocity indicated by the narrow $\mathrm{CO}$ component $\left(\sim 1 \mathrm{~km} \mathrm{~s}^{-1}\right)$, although it is lower than the one of the fast wind $\left(\sim 11 \mathrm{~km} \mathrm{~s}^{-1}\right)$ as traced by the broad CO component. Such a seemingly contradictory behavior may be due to the velocity field close to the stellar photosphere, which is dominated by shocks generated by the stellar pulsation. In fact, the high velocities indicated by the $\mathrm{SiO}$ maser line as compared to the (much) lower velocity indicated by the narrow $\mathrm{CO}$ line component is reminiscent of other pulsating AGB stars (see, e.g., Cernicharo et al. (1997); Herpin et al. (1998), Paper I), notably for the case of $\mathrm{L}^{2}$ Pup, where the effect is extreme. In Winters et al. (2002), we interpreted this behavior by means of the velocity field around a pulsating AGB star as it results from hydrodynamical modeling of a dust-forming circumstellar shell that produces only small amounts of dust. In that case, the wind is largely supported by the stellar pulsation, and radiation pressure on dust only weakly accelerates the gas beyond the sonic region. 
We do not find evidence of a detached shell, such as those found around some carbon stars by Olofsson et al. (1996), but rather find evidence of a succession of events that are shortspaced, $\sim 5^{\prime \prime}$ or $\left.1 \times 10^{16} \mathrm{~cm}\right)$. The timescale between 2 successive events thus might well be $\sim 300$ years. This is comparable to (possibly longer than) the timescale observed by Mauron \& Huggins (2000) in the shell of the carbon star IRC+10216. This kind of structure is reminiscent of the behavior found by Winters et al. (2000) for their theoretical $A B$ models, which show large variations in the mass loss rate on a timescale of a few $10^{2}$ years. These variations could produce a layered density structure similar to what is observed in the extended circumstellar shells of some pulsating red giants.

Finally, the model of Schröder et al. (1999) that produces only one single event of enhanced mass loss triggered by a thermal pulse for stars with initial masses $M_{\text {in }} \leq 1.3 M_{\odot}$ probably does not apply to EP Aqr. This would be consistent with the Dumm \& Schild (1998) estimate of the present EP Aqr mass of $1.7 M_{\odot}$.

We also note that Technetium, whose presence in a stellar atmosphere is a sign of a recent thermal pulse followed by a dredge-up event, was not detected by Lebzelter \& Hron (1999).

\section{Conclusions}

The ${ }^{12} \mathrm{CO}(2-1)$ and (1-0) emission from EP Aqr has been mapped with a spatial resolution of $\sim 1^{\prime \prime}$ and $2^{\prime \prime}$, respectively, and a spectral resolution of $0.1 \mathrm{~km} \mathrm{~s}^{-1}$. These maps were obtained by combining interferometric data obtained at the Plateau de Bure interferometer with short-spacing data obtained at the $30 \mathrm{~m}$ telescope. At our spatial resolution $\left(\sim 1^{\prime \prime}\right.$, or $\left.2 \times 10^{15} \mathrm{~cm}\right)$, the 2 spectral components seen in the single-dish line spectra were not observed to come from clearly different regions.

The images are largely circularly symmetric and show a smooth variation with velocity. The $\mathrm{CO}$ brightness distribution also is a smooth function of radius. However, on the $\mathrm{CO}(2-1)$ azimuthal average, two excesses can be seen at about $5^{\prime \prime}$ and $10^{\prime \prime}$ from the map center.

We do not find evidence of a bipolar structure in EP Aqr. We instead find that our data agree with a spherically-symmetric (but possibly clumpy) mass loss and that the Winters et al. (2000) $A B$ models may offer a good representation of the mass loss from the oxygen-rich, semi-regular variable EP Aqr.

Acknowledgements. We are grateful to IRAM Plateau de Bure staff and to Frédéric Damour at IRAM Granada for their support in the observations. The anonymous referee is thanked for her or his comments that helped to improve the presentation of the material. This research made use of the SIMBAD database, operated at CDS, Strasbourg, France, and of NASA's Astrophysics Data System.

\section{References}

Beichman, C. A., Neugebauer, G., Habing, H. J., Clegg, P. E., \& Chester, T. J. 1988, IRAS catalogues and atlases, Version 2, Explanatory supplement, NASA RP-1190 (NASA)

Boboltz, D. A., Diamond, P. J., \& Kemball, A. J. 1997, ApJ, 487, L47

Cernicharo, J., Alcolea, J., Baudry, A., \& González-Alfonso, E. 1997, A\&A, 319, 607

Dumm, T., \& Schild, H. 1998, New Astron., 3, 137

Fouqué, P., Le Bertre, T., Epchtein, N., Guglielmo, F., \& Kerschbaum, F. 1992, A\&AS, 93, 151

Glassgold, A. E., \& Huggins, P. J. 1983, MNRAS, 203, 517

González Delgado, D., Olofsson, H., Kerschbaum, F., et al. 2003, A\&A, 411, 123

Greenhill, L. J., Colomer, F., Moran, J. M., et al. 1995, ApJ, 449, 365

Herpin, F., Baudry, A., Alcolea, J., \& Cernicharo, J. 1998, A\&A, 334, 1037

Hogerheijde, M. R., \& van der Tak, F. F. S. 2000, A\&A, 362, 697

Jura, M., \& Kahane, C. 1999, ApJ, 521, 302

Jura, M., Kahane, C., \& Omont, A. 1988, A\&A, 201, 80

Jura, M., Chen, C., \& Plavchan, P. 2002, ApJ, 569, 964

Kahane, C., \& Jura, M. 1996, A\&A, 310, 952

Kastner, J. H. 1992, ApJ, 401, 337

Kemper, F., Stark, R., Justtanont, K., et al. 2003, A\&A, 407, 609

Knapp, G. R., \& Morris, M. 1985, ApJ, 292, 640

Knapp, G. R., Young, K., Lee, E., \& Jorissen, A. 1998, ApJS, 117, 209

Le Bertre, T., \& Gérard, E. 2004, A\&A, 419, 549

Lebzelter, T., \& Hron, J. 1999, A\&A, 351, 533

Matthews, L. D., \& Reid, M. J. 2007, AJ, 133, 2291

Mattsson, L., Höfner, S., \& Herwig, F. 2007, A\&A, 470, 339

Mauron, N., \& Huggins, P. J. 1999, A\&A, 349, 203

Mauron, N, \& Huggins, P.J. 2000, A\&A, 359, 707

Moreno, H., \& Carrasco, G. 1986, A\&AS, 65, 33

Nakashima, J.-I. 2006, ApJ, 638, 1041

Neri, R., Kahane, C., Lucas, R., Bujarrabal, V., \& Loup, C. 1998, A\&AS, 130, 1

Ossenkopf, V., \& Henning, T. 1994, A\&A, 291, 943

Olofsson, H., Bergman, P., Eriksson, K., \& Gustafsson, B. 1996, A\&A, 311, 587

Olofsson, H., Bergman, P., Lucas, R., et al. 2000, A\&A, 353, 583

Olofsson, H., González Delgado, D., Kerschbaum, F., \& Schöier, F. L. 2002, A\&A, 391, 1053

Pety, J. 2005, in SF2A-2005: Semaine de l'Astrophysique Française, ed. F. Casoli, T. Contini, J. M. Hameury, \& L. Pagani, 721

Schröder, K.-P., Winters, J. M., \& Sedlmayr, E. 1999, A\&A, 349, 898

Schöier, F. L., van der Tak, F. F. S., van Dishoeck, E. F., \& Black, J. H. 2005, A\&A, 432, 369

Teyssier, D., Hernandez, R., Bujarrabal, V., Yoshida, H., \& Phillips, T. G. 2006, A\&A, 450, 167

Winters, J. M., Le Bertre, T., Jeong, K. S., Helling, C., \& Sedlmayr, E. 2000, A\&A, 361, 641

Winters, J. M., Le Bertre, T., Nyman, L.-Å.., Omont, A., \& Jeong, K. S. 2002, A\&A, 388, 609

Winters, J. M., Le Bertre, T., Jeong, K. S., Nyman, L.-Å., \& Epchtein, N. 2003, A\&A, 409, 715 (Paper I)

Wittkowski, M., Boboltz, D. A., Ohnaka, K., Driebe, T., \& Scholz, M. 2007, A\&A, 470, 191 